\title{
Diseño de Actividades para el Aprendizaje en la Asignatura de Construcción 2 del Grado en Fundamentos de la Arquitectura
}

\section{Activities Design for Learning in Construction 2 Subject to Degree in Architecture}

BEgOÑA BLANDÓN GONZÁLEZ

ORCID: https://orcid.org/0000-0003-1025-5675 Universidad de Sevilla

Escuela Técnica Superior de Arquitectura (ETSA) Departamento de Construcción Arquitectónica 1 bblandon@us.es

Fecha de recepción: 23-06-2019

Fecha de aceptación: 02-07-2019

DOI: http://dx.doi.org/10.12795/9788447221912.045

Pp.: 1024-1052 


\section{Resumen}

El diseño de los Ciclos de Mejora que se aplican en el aula (CIMA) como parte de la formación del profesorado de la Universidad de Sevilla está permitiendo el estudio de cada asignatura protagonista desde una triple vertiente: los contenidos a impartir en el aula, la metodología docente utilizada en su aplicación y la evaluación de la progresión del aprendizaje experimentado en el estudiante.

La asignatura de Construcción 2 ha sido objeto de este CIMA por tercer año consecutivo, lo que está permitiendo profundizar con distintos enfoques docentes dentro y fuera del aula. En el curso 2019, y continuando con el objetivo de dinamizar la docencia e implicar al estudiante en su propio aprendizaje, se buscan alternativas que despierten su interés y lo acerque a su futura práctica profesional. Para ello, se elaboran actividades que faciliten el aprendizaje de los conocimientos teóricos y prácticos que la asignatura requiere. La sistemática seguida ha permitido obtener interesantes resultados y abrir nuevas perspectivas en la mejora de la docencia y el aprendizaje de la asignatura de Construcción 2.

Palabras Clave: CIMA, Experimentación Docente Universitaria, Aprendizaje por problemas, Construcción, Arquitectura.

Jornadas de Formación e Innovación Docente del Profesorado | № 2 (2019) Esta obra se distribuye con la licencia Creative Commons 


\section{Abstract}

The design of the Improvement Cycles in Classroom (ICIC) as part of the teacher formation of the University of Seville is allowing the study of each main subject from a triple perspective: the contents to be taught in the classroom, the teaching methodology employed in its application and the evaluation of the progression of the learning experienced by the student.

The Construction 2 subject has been the object of this ICIC for the third consecutive year, which is enabling in-depth study with different teaching approaches inside and outside the classroom. In the academic course 2019, and continuing with the objective of dynamising teaching and involving students in their own learning, alternatives are being sought that awaken their interest and bring them closer to their future professional practice. To this end, activities are developed to facilitate the learning of the theoretical and practical knowledge that the subject requires. The systematics followed have allowed to obtain interesting results and to open new perspectives in the improvement of the teaching and the learning of the subject of Construction 2.

Keywords: ICIC, University Teaching Innovation, Learning by problems, Construction, Architecture.

Jornadas de Formación e Innovación Docente del Profesorado | № 2 (2019) Esta obra se distribuye con la licencia Creative Commons 


\section{Introducción}

En 2017, con motivo del Curso General de Docencia Universitaria y como parte del Programa de Formación e Innovación Docente del Profesorado (FIDOP) organizado por el Instituto de Ciencias de la Educación (ICE) que la Universidad de Sevilla pone a disposición del docente, nace el interés por aportar un nuevo diseño en la docencia de la asignatura de Construcción 2 perteneciente al Grado en Fundamentos de la Arquitectura. Esta propuesta permitiría transformar el desarrollo tradicional de las clases magistrales impartidas hasta entonces, en un formato de clases más dinámico en las que el estudiante y docente interactuaran (Porlán, 2017). Los resultados obtenidos en las sesiones en las que se prueba un primer diseño impulsan este proyecto planteando su extensión a toda la asignatura.

La asignatura de Construcción 2, sobre la que se desarrolla el CIMA, pertenece al Departamento de Construcciones Arquitectónicas 1 de la Escuela Técnica Superior de Arquitectura de la Universidad de Sevilla. Esta asignatura se imparte en 2o curso del Plan de Estudios 2012 y consta de 6 créditos ECTS de formación obligatoria. La línea de contenidos que desarrolla se centra en el diseño de los sistemas constructivos: Estructuras, Cimientos y las Envolventes del edificio (definiendo materiales y productos correspondientes en cada caso y un primer acercamiento al cálculo y pre dimensionado de elementos). 


\section{BEgOÑA BLANDÓN GONZÁLEZ}

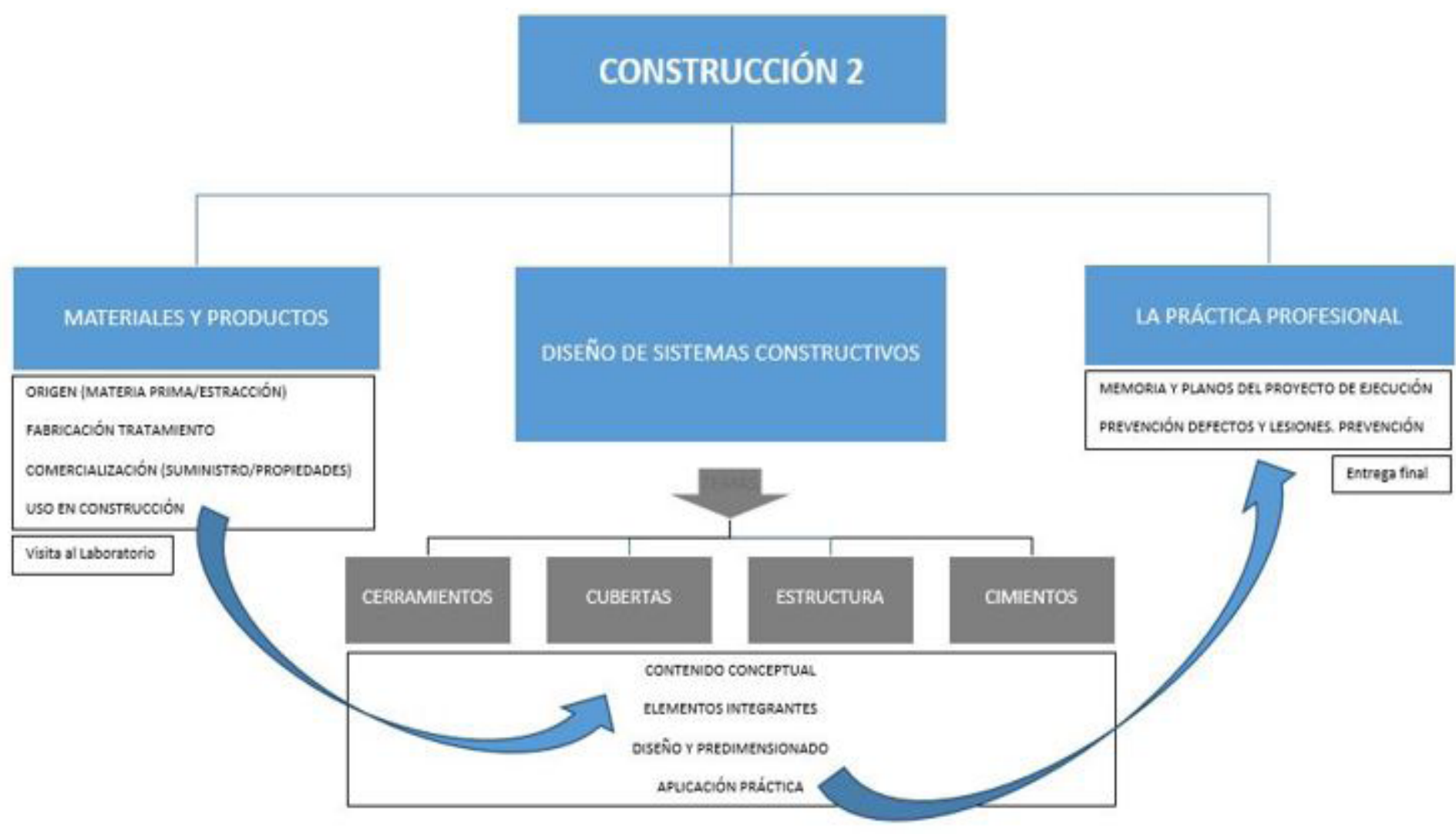

Figura 1. Mapa de Contenidos de la asignatura Construcción 2. Fuente: CIMA 2018 (Autor)

Jornadas de Formación e Innovación Docente del Profesorado | № 2 (2019) Esta obra se distribuye con la licencia Creative Commons

Reconocimiento-NoComercial-SinObraDerivada 
Asimismo, la asignatura de Construcción 2 ofrece, en primera convocatoria, dos opciones como parte del sistema de evaluación del aprendizaje del estudiante: la opción tradicional de un examen final completo y, como alternativa, la evaluación "continua" de conocimientos. En este segundo caso, la calificación es resultante de distintos apartados y porcentajes:

Tabla 1. Distribución de porcentajes en el sistema de evaluación continua. Fuente: Autor.

\begin{tabular}{|c|c|c|c|c|}
\hline $\begin{array}{c}\text { ASISTENCIA } \\
(\mathbf{8 0} \% \text { mínimo })\end{array}$ & $\begin{array}{c}\text { PRÁCTICA DE } \\
\text { CLASE }\end{array}$ & PRÁCTICA DE CURSO & $\begin{array}{c}\text { PRUEBA } \\
\text { ESCRITA }\end{array}$ & $\begin{array}{c}\text { CALIFICACIÓN } \\
\text { FINAL }\end{array}$ \\
\hline $5 \%$ & $35 \%$ & $40 \%$ & $20 \%$ & $100 \%$ \\
\hline $\begin{array}{c}\text { Este aspecto incluye la } \\
\text { implicacion y participación } \\
\text { del estudiante en el aula. }\end{array}$ & $\begin{array}{c}\text { Actividades del aula, } \\
\text { visita al laboratorio, } \\
\text { etc. }\end{array}$ & $\begin{array}{c}\text { Proyecto de Ejecución } \\
\text { desarrollado en casa y entregado } \\
\text { a final del cuatrimestre. }\end{array}$ & $\begin{array}{c}\text { Prueba/control } \\
\text { de } \\
\text { conocimientos. }\end{array}$ & $\begin{array}{c}\text { Nota correspondiente a } \\
\text { la primera convocatoria }\end{array}$ \\
\hline
\end{tabular}

En el curso 2018, durante la Fase de Permanencia del Programa de Formación e Innovación Docente del Profesorado y como parte de la Red Permanente de Formación (REFID), se avanza sobre el diseño de la asignatura completa aportando un Ciclo de Mejora en el Aula (CIMA) en el que se ordenan y jerarquizan los contenidos del temario de la asignatura de Construcción 2, se revisa el nuevo modelo metodológico iniciado en el curso anterior y se prevén distintas actividades en el aula que, a partir de una secuencia inicial de preguntas, tratan de despertar el interés del estudiante sobre la materia que se imparte (Blandón, 2018). A partir de la aplicación en el aula del CIMA propuesto, se anotan los aciertos y mejoras percibidas en el aprendizaje de la materia a fin de consolidarlos. Sin embargo, respecto a las dificultades encontradas, se observa que la mayor parte de ellas, se localizan en las propias actividades desarrolladas en el aula. A este respecto:

- Las actividades desarrolladas no se acompañaron de un enunciado escrito. Se fueron dictando datos previos e información adicional a medida que se demandaba por parte del estudiante. Como consecuencia, resultaron excesivas las intervenciones del profesor durante el desarrollo del trabajo interrumpiendo continuamente 
para aclaraciones o repeticiones. Se detectó que los estudiantes no trabajaron su autonomía.

- Se observó la necesidad de uso de la normativa en las actividades de cálculo (requerían de tablas y fórmulas). Para evitar retrasos en la búsqueda de las mismas por parte del estudiante, se proyectaron pantallazos de los P. Point de la asignatura. Esta dinámica resultó muy desordenada en el aula.

- El desarrollo de las escaleras de aprendizaje al finalizar el CIMA fue dificil resultando incompleto y confuso por lo que, no reflejó su utilidad a fin de conocer la progresión del aprendizaje.

- Respecto a la entrega de la Práctica de Curso que el estudiante debe aportar a final del cuatrimestre como parte de la evaluación continua, se detectó la dificultad del estudiante para elaborar la Memoria Descriptiva y Justificativa como parte del Proyecto de Ejecución.

Con vistas al curso 2019 y, a partir de las carencias detectadas en el curso anterior, se decide profundizar y mejorar el diseño de las actividades desarrolladas en el aula concretando la definición de problemas (Bur, 2012) cuya resolución garantice el aprendizaje previsto. En esta dirección se proponen nuevas actuaciones y un nuevo diseño de CIMA basadas en unos principios didácticos en los que se busca:

- Mantener la secuencia de preguntas (Bain, 2008) como curiosidades previas en el formato de clases a fin de generar el desarrollo posterior de las actividades: ¿Para qué sirve?, ¿Qué elementos lo integran?, ¿Cómo los diseño?, ¿Me interesa en mi práctica profesional?

- Aumentar el desarrollo de actividades prácticas y resolución de problemas en el aula.

- Diseñar las actividades que promuevan la autonomía del estudiante en su resolución (Finkel, 2008).

- Impulsar clases que más interactivas buscando la participación del estudiante en el aula. 
- Reducir la línea individualista del aprendizaje e incluir actividades en grupo con la puesta en común de los resultados.

- Facilitar al estudiante el conocimiento sobre su evolución y la progresión en su aprendizaje. En cada actividad se incorporará la posibilidad de autoevaluación. A final de cada ejercicio el estudiante debiera conocer el nivel en el que se encuentra y ser consciente de su evolución.

\section{Objetivo propuesto}

El diseño y desarrollo del CIMA propuesto para el curso 2019 se llevará a cabo en la asignatura de Construcción 2, completando el trabajo ya iniciado en el curso anterior y ordenando el desarrollo de las actividades. Las mejoras propuestas, reflexionarán sobre la identidad de los contenidos, la metodología más adecuada para su aprendizaje y la evaluación de los conocimientos adquiridos. En esta dirección, se definirán los enunciados de las actividades para centrar la disposición del profesor como tutor de las mismas y favorecer un aprendizaje más autónomo sin continuas interrupciones durante el desarrollo de los ejercicios.

\section{Diseño experimental propuesto}

Tal y como se plantea, en el diseño de la docencia se recurre a un modelo que permitirá una concepción de clases más dinámicas. El aprendizaje se propone con distintas actividades de carácter variado y la resolución de problemas en el aula que implican una mayor participación del estudiante. En este proceso, se opta por acercar al estudiante a la realidad de su práctica profesional por lo que, cada una de las actividades propuestas van surgiendo con la necesidad de avanzar en el diseño de un Proyecto de Ejecución completo (como parte del Proyecto de Edificación de viviendas en el que trabajan durante el cuatrimestre). 
BEGOÑA BLANDÓN GONZÁLEZ
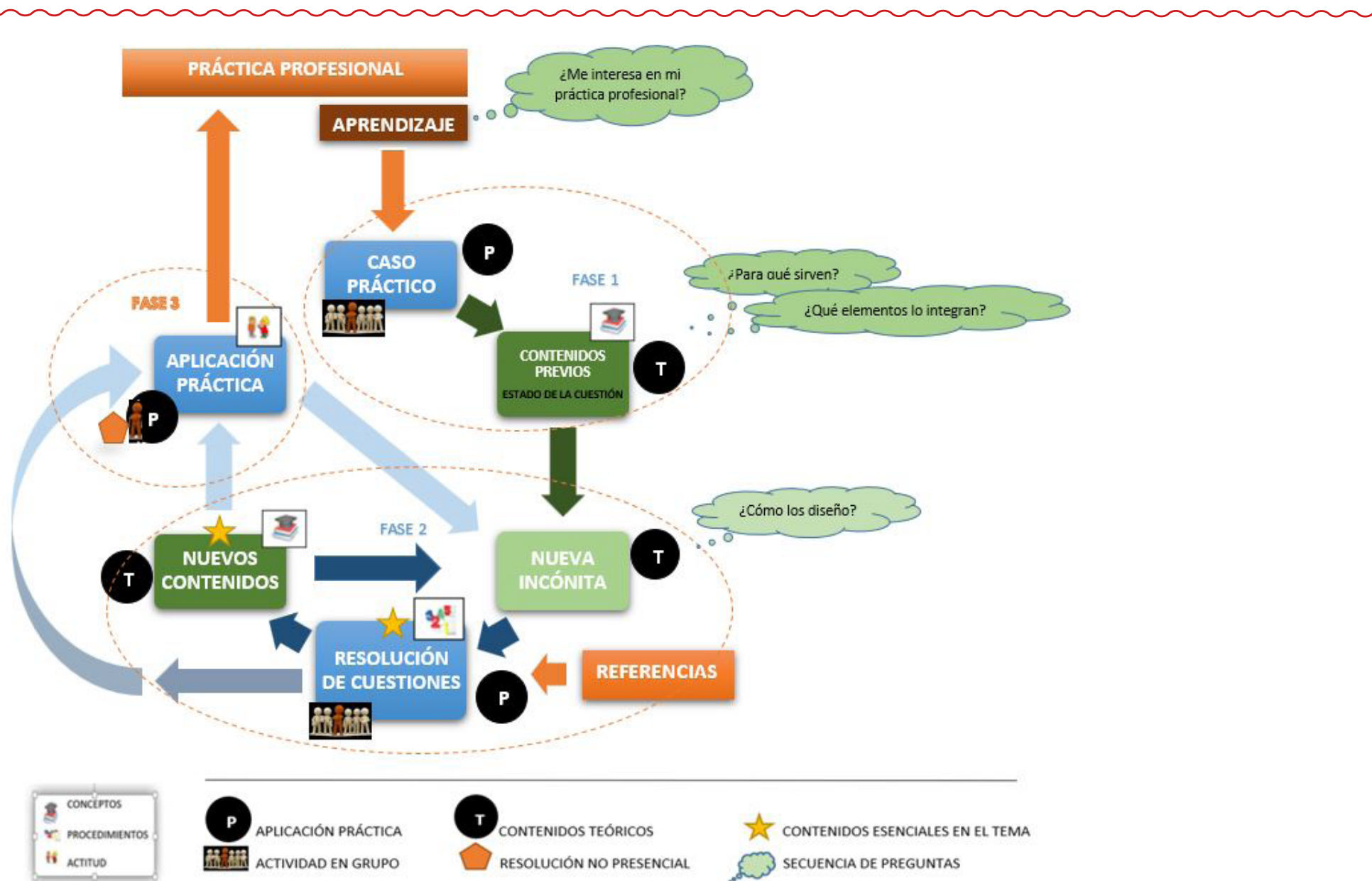

th CONTENIDOS ESENCIALES EN EL TEMA

Figura 2. Modelo metodológico y desarrollo en el aula. Autor

Jornadas de Formación e Innovación Docente del Profesorado I № 2 (2019) 
A este respecto, la aplicación en el aula de este modelo metodológico requiere un mayor esfuerzo de atención por parte del estudiante y una mayor organización de la docencia por parte del profesor. Así, el aprendizaje en el aula se desarrolla de forma más autónoma lo que implica que, la figura del profesor, pasa a ser únicamente la de tutor del proceso. En este caso, ambos comparten la responsabilidad de su aprendizaje.

\section{Estructura y contenido de las actividades propuestas}

Reforzando la idea de alcanzar un aprendizaje más autónomo durante el desarrollo de las actividades del aula, aunque se elaboran fichas independientes para cada ejercicio/tema, se diseña un formato único que trata de dar uniformidad a la propuesta.

Jornadas de Formación e Innovación Docente del Profesorado | № 2 (2019) Esta obra se distribuye con la licencia Creative Commons 


\section{BEGOÑA BLANDÓN GONZÁLEZ}

\section{CUESTIONARIO DE INICIO DE CURSO}

OвJеIVO: ACTIMDAD O

Con esta actividad se pretende conocer el nivel de conocimientos conceptuales y procedimentales con los que el estudiante se incorpora a la asignatura de Construcción 2 (en $2^{\circ}$ curso del Grado en Fundamentos de la Arquitectura). Asimismo, como cuestionario inicial al desamollo del curso, trotorád de recuperar y ordenen
los conocimientos aprendidos en el curso anterior y despertar el interés por la utilidad de la asignatura.

CONCEPTOS QUE DEBES CONTROLAR ANTES DEL INICIO DE LA ACTIVIDAD:

¿¿Cuáles la misión de los elementos estructurales en el edificio?

¿Como se dirigen las cargos en un forjado unidireccional?
¿Quét tipo de cimentación directa recibe la carga de muros, y pilares?

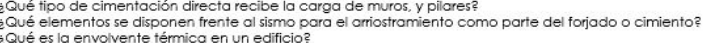

ENUNCIADO:

Sobre las plantas que se aporton representa los siguientes datos:
En planta baja:

(1)

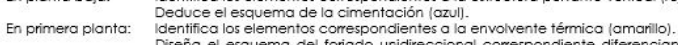

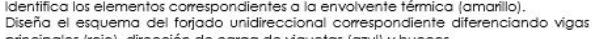

Dibuja las dos secciones básicas señalodas desde la cubiertio hasto el cimiento diferenciando envolvente y
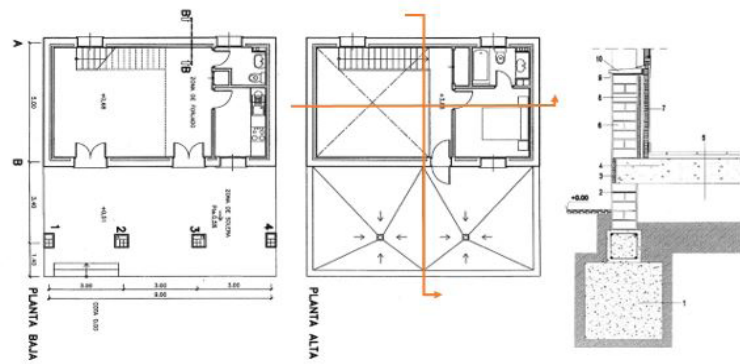

Para la sección odjunta, indica el nombre, material y aimensiones aproximados de los elementos
representodos, dibuja el contenido del forjado sanitorio y confirma su posible punto de corte en la planto. DATOS DE PARTIDA:

Los conocimientos del estudiante
Fundamentos de la Arquitectura).

ACTVIDAD INDIVIDUAL $\quad$ (Thempo previsto: 45 min)
CUESIONARIO DE INICIO DE CURSO

ACTMIDAD O

METODOLOGÍA DE TRABAJO Y RECOMENDACIONES: (no se aporta al estudiante)

10. Con los datos que se aportan, dibuja en perspectiva la volumetriá del edifico. Con esto te aseguras
entender bien su geometría y niveles. Si es necesario, esboza un par de secciones básicas en las das direcciones.

$2^{\circ}$. Identifica en ambos plantos los posibles elementos portantes verticales (muros de carga y pilares).
Recuerda que, en ambos casos, debe corresponderse su ubicación en ambas plantas para posibilitar la

3․ Compruebo dimensiones entre apoyos $y$, para los casos más favorables (menor Iuz, huecos reducidos en muros, etc) deduce los ejes de corga (puedes reflejarlo con inea discontinua). crada vano.
Finalmente compreveba que todas las viguetas previstas apoyan sobre esas vigas. Si es necesario, incluye
vigas brochales (revisa el caso del hueco de escolera).

$4^{0}$. Representa las zapotas aisladas y corridas bajo pilares y muros arriostrando con las comespondientes vigas (combina zapatos si es necesario). Cuida los peldaños de acceso o plataformas y porches en
edificio asegurando que se apoyan sobre un elemento estable y no directamente sobre el terreno. 50. Señala la envolvente térmica del edificio. Recuerda que debe cerrorse separando el exterior del
espacio interior habitado (los puentes térmicos quedarón dentro de esa envolvente). $6^{\circ}$. A partir de los datos anteriores, levanto las secciones marcodas sin mós definición que distinguir los

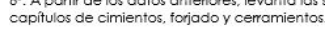

$77^{\circ}$. Observa los elementos envolventes y el cimiento que aparece en la sección. Si observas la planta baja. la sección BB' no incluye la ventana. Señala el lugar de corte en planta y especifica cada uno de sus RESULIADOS Y DISCUSIÓN

Puesta en común de resultados obtenidos.

(a)

NIVEL DE APRENDIZAJE OBTENIDO POR EL ESTUDIANTE

Puntúa en las verticales de los peldoños el nivel de acierto con el que hos superado cada ejercicio de la actividad (el nivel más olito se corresponde con el dominio en la materia y, el más bojo. al desconocimiento muchas incógnitas).

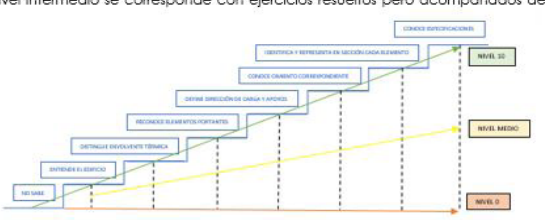

INCIDENCIAS EN EL DESAPROUO DE LAACTIVIDAD

¿Cómo mejoraros esta actividad?

¿̇En qué punto de la actividad has encontrado dificultades?

ACTIVIDAD INDIVIDUAL_ (Tiempo previsto: 45 min)

Figura 3. Ejemplo de formato previsto para desarrollo de actividades en el aula. Autor.

Jornadas de Formación e Innovación Docente del Profesorado | № 2 (2019) 
Persiguiendo los principios didácticos mencionados, este formato incluye distintos apartados que informan al estudiante sobre el número de participantes, el tiempo previsto para su resolución o aquellos aspectos que puedan ordenar la materia y su resolución, así como las conclusiones del aprendizaje que se pretende:

- Objetivos a alcanzar con el desarrollo de la actividad propuesta. En este apartado se distingue su finalidad como propuesta de repaso de conocimientos o como recurso para la adquisición de nuevos contenidos, ya sean conceptuales, procedimentales y/o actitudinales. A este respecto, se pueden diferenciar actividades más reflexivas (que suponen un punto de partida para nuevos contenidos y cuyo desarrollo se acompañan de una secuencia de preguntas que lo guían).

- Listado de conocimientos (conceptuales y procedimentales) que el estudiante debe controlar al inicio de la actividad.

- Enunciado de la actividad (incluyendo, de forma muy clara, los distintos apartados que se deben resolver) e imágenes, planos o detalles del edificio a escala suficiente sobre la que se trabaja. El formato entregado permite su resolución (a mano) sobre el mismo apoyando la expresión gráfica del estudiante.

- Datos de partida sin los cuales el estudiante no puede resolver la actividad (apuntes, material de dibujo o cálculo). Se aportan las tablas y figuras correspondientes a la normativa de aplicación.

- Metodología/fases de trabajo requeridas como guía para el desarrollo adecuado de cada actividad evitando la continua atención que requieren del profesor.

- Discusión de resultados obtenidos y puesta en común (con espacio y guion para las posibles alternativas por parte de otros compañeros). En este apartado se pueden plantear nuevas cuestiones de interés y la introducción a la siguiente actividad prevista.

- Escaleras de progresión del aprendizaje con vistas a un posible seguimiento que permita al estudiante 
conocer el nivel en el que se encuentra al desarrollar la actividad. Como sistemática a seguir para facilitar su autoevaluación, se propone reflejar su posición en gráficos de barras (específicos de cada actividad) que llevan a configurar una escalera y le permiten posicionarse en relación con el nivel que debe adquirir.

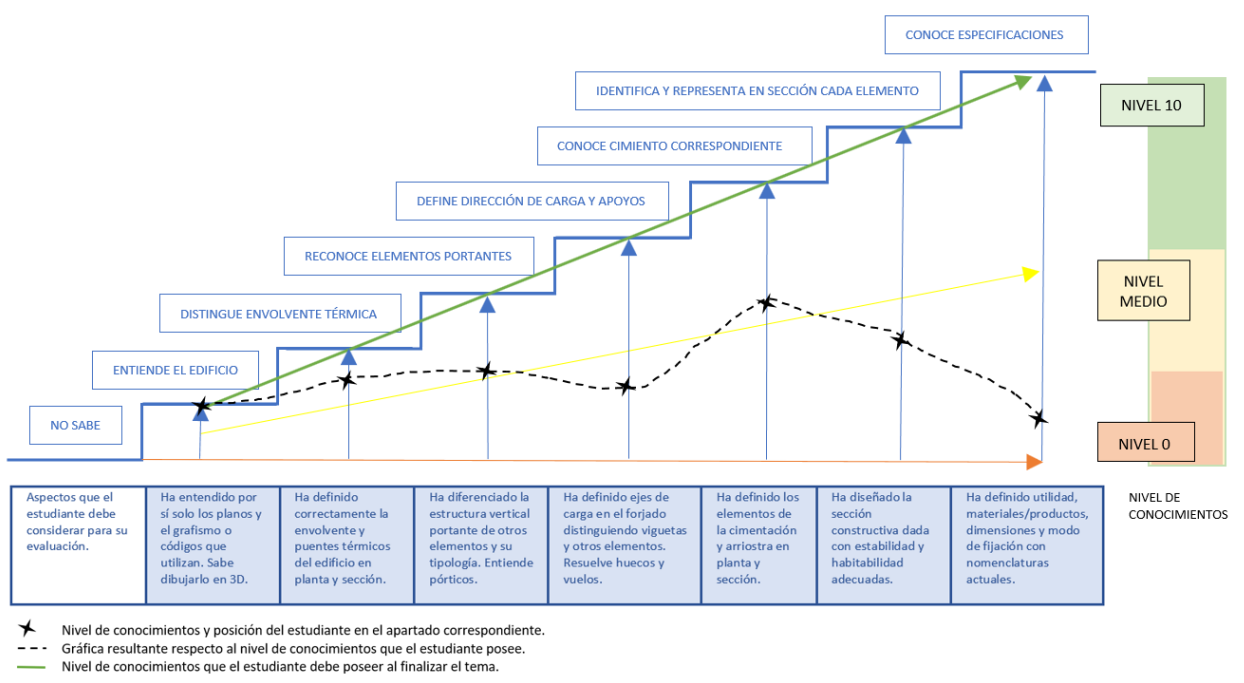

Figura 4. Ejemplo de autoevaluación del aprendizaje propuesto. Autor.

Mini cuestionario de evaluación de la actividad propuesta. Tras realizar el ejercicio el estudiante puede indicar las incidencias o dificultades que ha detectado durante el desarrollo de la actividad a fin de revisar el procedimiento y aportar mejoras en el diseño.

En cualquier caso, cada una de las actividades se apoya en la anterior y origina nuevas cuestiones que enlazan con la siguiente tratando de mantener cierta continuidad y relación con el proceso de la práctica profesional. 


\section{Diseño de las sesiones propuestas}

En el actual plan de estudios, las clases de Construcción 2 se organizan en un horario que centra la docencia de la asignatura en un solo día de la semana, con una duración de 4 horas. Al objeto de evitar el aburrimiento que acompaña al cansancio como consecuencia de una jornada tan extensa, el desarrollo de cada una de las sesiones en el aula, se propone diferenciando tres etapas o fases de trabajo:

Fase 10- Espacio y tiempo destinado al Repaso de Conocimientos ( \pm 60 minutos):

En la primera sesión de cada nuevo tema, se reparte una Prueba de Inicio a fin de conocer los conocimientos con los que el estudiante se incorpora, esta prueba incluye casos a estudio sobre uno o varios edificios existentes (se agrupan mesas - equipos de 2-3 estudiantes) tratando de reconocer aquellas soluciones constructivas correspondientes al tema y cuya revisión de errores o consulta de dudas durante el desarrollo de la actividad facilita el repaso de los conocimientos básicos adquiridos en primer curso (Construcción 1) y que suponen la base del nuevo curso.

En el resto de sesiones del tema, esta primera parte se destina la revisión y supervisión de los trabajos encargados en la sesión anterior (llamamos Prácticas 1,2,3) como parte del aprendizaje no presencial. Desde la tarima, en la pizarra, se comentan los errores más repetidos, se repasan criterios de corrección y resuelven dudas generales surgidas durante el aprendizaje no presencial. El estudiante, de forma individual, corrige su práctica (detectando sus propios errores). Posteriormente, de forma individualizada, por las mesas se resuelven las dudas o preguntas particulares que hayan quedado y se califica el trabajo. 
Fase 20- Secuencia de Preguntas y

resolución ( \pm 90 minutos):

A partir del repaso previo inicial surgen incógnitas que se resuelven en el aula a partir de actividades de las que, durante su desarrollo y/o conclusión (15-30 minutos- grupos de 2-3 estudiantes), permiten la reflexión y extracción de los contenidos esenciales de la asignatura en este punto (llamamos Actividades 1,2,3). La puesta en común de los resultados y la exposición de nuevos casos por parte del estudiante, deriva en nuevas cuestiones de interés y permite disponer de una casuística variada sobre distintas soluciones que acercan su formación a la futura práctica profesional.

En esta etapa se profundiza en los contenidos teóricos esenciales de la asignatura (aspectos de pre dimensionado y armado de las soluciones previstas, exigencias de diseño que garantizan la estabilidad, habitabilidad, así como la configuración constructiva del elemento y su encuentro con forjados, pilares, cubierta, el terreno, etc. soluciones más comunes y los materiales/productos empleados).

\section{Fase 3o- Aplicación Práctica y Práctica Profesional ( \pm 90 minutos):}

Extraídos y expuestos los contenidos esenciales del tema de forma grupal, el estudiante debe enfrentarse a ellos de forma individual con el desarrollo de la Práctica de Curso (Prácticas 1,2,3). Esta actividad se inicia en el aula ( \pm 30 minutos) y puede finalizarse en casa dejando pendiente su entrega en la semana posterior.

\section{Aplicación en el aula del CIMA}

El diseño experimental de las actividades propuestas se aplica en el aula durante 38 horas que se reparten en 9 
sesiones de 4 horas y 2 horas independientes que se extraen de la primera y última clase del curso tal y como se indica en la programación aportada. Durante el curso 2019, el nuevo diseño experimental se llevará a cabo sobre los temas de introducción a la estructura, cerramientos, cubiertas y forjados y cimientos.

Tabla 2. Programación de actividades de clase previstas facilitada a los estudiantes. Autor.

\begin{tabular}{|c|c|c|c|c|c|c|}
\hline $\begin{array}{l}\text { FECHA } \\
2019\end{array}$ & TEMARIO & $\begin{array}{l}\text { CONTENIDO DE } \\
\text { LA DOCENCIA }\end{array}$ & & ACTIVIDADES DEL AULA & & ACTIVIDADES NP \\
\hline \multirow{3}{*}{11 FEB } & & & & PRESENTACIÓN DEL CURSO & & P0. PRESENTACTÓN P.CURSO \\
\hline & & & $\begin{array}{c}\text { PASE } \\
1\end{array}$ & $\begin{array}{l}\text { ACT } 0 \text {. CUESTIONARIO DE INICIO } \\
\text { A LA ASIGNATURA. }\end{array}$ & & \\
\hline & EL REPL.ANTEO & $\begin{array}{l}\text { REPASO DE } \\
\text { CONOCIMIENTOS } \\
\text { RECORRIDO } \\
\text { FOTOGRAFICO }\end{array}$ & $\begin{array}{l}1 \\
2\end{array}$ & $\begin{array}{l}\text { ACT R1. LA IMPORTANCIA DEL } \\
\text { REPL.ANTEO. } \\
\text { ACT R2. DATOS DE INTERES. }\end{array}$ & & $\begin{array}{l}\text { RECUPERACIÓN DE } \\
\text { PRACTICAS Y APUNTES Cl }\end{array}$ \\
\hline $\begin{array}{l}2 \\
\mathrm{FFBH}\end{array}$ & $\begin{array}{l}\text { SISTEMAS } \\
\text { CONSTRUCTIVOS }\end{array}$ & $\begin{array}{l}\text { MATERIALES Y } \\
\text { PRODUCTOS }\end{array}$ & & VISITA AL LABORATORIO & & \\
\hline $\begin{array}{ll}3 \\
2 S \mathrm{FFB}\end{array}$ & \multirow[t]{2}{*}{$\begin{array}{l}\text { ESTRUCTURAS DE } \\
\text { H.A. }\end{array}$} & $\begin{array}{l}\text { RECORRIDO } \\
\text { FOTOGRÁFICO. } \\
\text { EXIGENCIAS } \\
\text { NORMATIVAS. }\end{array}$ & $\begin{array}{c}1 \\
1-2\end{array}$ & $\begin{array}{l}\text { ACT E1. REPASO DE } \\
\text { CONOCIMIENTOS. } \\
\text { ACT E2. ESQUELETO } \\
\text { ESTRUCTURAL. }\end{array}$ & $\begin{array}{c}\text { FASE: } \\
3\end{array}$ & $\begin{array}{l}\text { P01.ESQUELETO } \\
\text { ESTRUCTURAL }\end{array}$ \\
\hline $\begin{array}{l}4 \mathrm{MAR} \\
4\end{array}$ & & NOMENCLATURAS & $\begin{array}{l}2 \\
2 \\
\end{array}$ & $\begin{array}{l}\text { ACT E3. ELEMIENTOS DE H.A. } \\
\text { ACT REV1. CLASE INVERTIDA* }\end{array}$ & 3 & $\begin{array}{l}\text { P.POINT: PR.ODUCTOS DE H.A } \\
\text { EXPOSICIÓN ACT REV1* }\end{array}$ \\
\hline 5 II MAR & \multirow[t]{2}{*}{$\begin{array}{l}\text { FORJADOS } \\
\text { UNIDIRECCIONALES } \\
\text { DE H.A }\end{array}$} & $\begin{array}{l}\text { RECORRIDO } \\
\text { FOTOGRÁFICO. } \\
\text { EXIGENCIAS } \\
\text { NORMATIVAS }\end{array}$ & 2 & $\begin{array}{l}\text { ACT F1. REPASO DE } \\
\text { CONOCIMIENTOS. } \\
\text { ACT F2. DISENOO DE SECCIONES } \\
\text { DE H.A. }\end{array}$ & & P. POINT FORJADOS $\mathrm{C} 1$ \\
\hline $\begin{array}{l}6 \\
18 \text { MAR }\end{array}$ & & $\begin{array}{l}\text { PROCESO DB } \\
\text { CÁLCULO }\end{array}$ & 2 & $\begin{array}{l}\text { ACT F3. PREDIMENSIONADO Y } \\
\text { ARMADO DE ELEMENTOS. } \\
\text { CANTO MIINIMO Y VIGUETAS }\end{array}$ & 3 & $\begin{array}{l}\text { PF1. REPLANIEO DE } \\
\text { FORJADOS } \\
\text { PF2. DETALLES FORJADO }\end{array}$ \\
\hline \multirow[t]{2}{*}{$\begin{array}{l}7 \\
25 \mathrm{MAR}\end{array}$} & ESCALERAS & $\begin{array}{l}\text { RECORRIDO } \\
\text { FOTOGRÁFICO. } \\
\text { EXIGENCIAS } \\
\text { NORMATIVAS. } \\
\end{array}$ & $\begin{array}{c}1 \\
1-2\end{array}$ & $\begin{array}{l}\text { ACT ES1. REPASO DE } \\
\text { CONOCIMIENTOS } \\
\text { ACT ES2. DISEÑO DE ESCALERA. }\end{array}$ & 3 & $\begin{array}{l}\text { PES1. DISENYO DB LA } \\
\text { ESCALBRA Y DETALLE DEL } \\
\text { PELDANOO }\end{array}$ \\
\hline & REVESTMMIENTOS & $\begin{array}{l}\text { PRODUCTOS Y } \\
\text { EJECUCION }\end{array}$ & 3 & $\begin{array}{l}\text { LA CLASE INVERTIDA*: } \\
\text { EXPOSICION REVESTIDOS } \\
\text { HORIZONTALES EN SUELOS }\end{array}$ & & $\begin{array}{l}\text { P.POINT: PRODUCTOS } \\
\text { CBRÁMICOS, MADERAS, } \\
\text { PETREOS Y OTROS. }\end{array}$ \\
\hline 8 & \multirow[t]{2}{*}{ CERRAMIENTOS } & $\begin{array}{l}\text { ESTABILIDAD. } \\
\text { EXIGENCIAS } \\
\text { NORMATIVAS Y } \\
\text { HABITABILIDAD }\end{array}$ & $1-2$ & $\begin{array}{l}\text { ACT C1. REPASO DE } \\
\text { CONTENIDOS. } \\
\text { ACT C2. ENVOL VENTES Y } \\
\text { PARTICIONES. }\end{array}$ & 3 & $\begin{array}{l}\text { P1CE. REPLANTEO DE LA } \\
\text { ALBANILERIA. } \\
\text { P2CE, TIPOLOGIA DE } \\
\text { CERRAMIENTOS } \\
\text { P. POINT: CERRAMIENTOS }\end{array}$ \\
\hline $\begin{array}{l}9 \\
8_{\text {ABR }}\end{array}$ & & $\begin{array}{l}\text { PUNTOS } \\
\text { SINGULARES }\end{array}$ & 2 & $\begin{array}{l}\text { ACT C3. LESIONES EN LOS } \\
\text { BNCUENTROS DE FACHADA }\end{array}$ & 3 & $\begin{array}{l}\text { P3CE. BIVCUENTROS DEL CTE } \\
\text { P. POINT: HABITABIIDAD Y } \\
\text { p. STNGULARES }\end{array}$ \\
\hline $\begin{array}{l}10 \\
22 \mathrm{ABR}\end{array}$ & REVESTMMIENTOS & $\begin{array}{l}\text { PRODUCTOS Y } \\
\text { EJECUCIÓN }\end{array}$ & 3 & $\begin{array}{l}\text { LA CLASE INVERTIDA*: } \\
\text { EXPOSICIÓN REVESTIDOS } \\
\text { VERTICALES }\end{array}$ & & $\begin{array}{l}\text { P.POINT: YBSO, CAL, } \\
\text { CEMENTO Y OTROS } \\
\text { PRODUCTOS. }\end{array}$ \\
\hline 119 & CUBIERTAS & $\begin{array}{l}\text { ELEMENTOS Y } \\
\text { TIPOS DE FALDÓN. }\end{array}$ & $\begin{array}{c}1 \\
1-2\end{array}$ & $\begin{array}{l}\text { ACT CU1. REPASO DE } \\
\text { CONOCIMIENTOS } \\
\text { ACT CU2. DISENO Y REPLANTEO }\end{array}$ & 3 & $\begin{array}{l}\text { P POINT: CUBIERTAS } \\
\text { PICU. TRAZADO EN PLANTA } \\
\text { CUBIERTAS }\end{array}$ \\
\hline \multirow[t]{2}{*}{$12 \mathrm{MAY}$} & & $\begin{array}{l}\text { PUNTOS } \\
\text { SINGULARES }\end{array}$ & $1-2$ & $\begin{array}{l}\text { ACT CU3. LESIONES EN LOS } \\
\text { ENCURNTROS DEL FALDÓN }\end{array}$ & 3 & $\begin{array}{l}\text { P2CU.ENCUENTROS FALDON } \\
\text { P. POINT: P. SINGGULARES }\end{array}$ \\
\hline & REVESTMMIENTOS & $\begin{array}{l}\text { PRODUCTOS Y } \\
\text { EJECUCIÓN }\end{array}$ & 3 & $\begin{array}{l}\text { LA CLASE INVERTIDA*: } \\
\text { EXPOSICIÓN REVESTIDOS } \\
\text { HORIZONTALES DE TECHOS }\end{array}$ & & $\begin{array}{l}\text { P.POINT: YESOS Y } \\
\text { PRODUCTOS DE ESCAYOLA. }\end{array}$ \\
\hline \multirow[t]{2}{*}{13} & $\begin{array}{l}\text { ACCIONES EN LA } \\
\text { EDIFICACION }\end{array}$ & $\begin{array}{l}\text { TIPOS DE } \\
\text { ACCIONES }\end{array}$ & $1-2$ & $\begin{array}{l}\text { ACT CIM1. ACCTONES } \\
\text { GRAVITATORIAS }\end{array}$ & 3 & $\begin{array}{l}\text { PISECC: SECCTON } \\
\text { CONSTRUCTIVA FACHADA }\end{array}$ \\
\hline & \multirow[t]{2}{*}{$\begin{array}{l}\text { TBRRENO Y } \\
\text { CIMIENTOS }\end{array}$} & $\begin{array}{l}\text { EL ESTUDIO } \\
\text { GEOTBCNICO } \\
\text { TIPOS CIMIENTOS. }\end{array}$ & 1 & $\begin{array}{l}\text { ACT CIM2. REPASO DE } \\
\text { CONOCIMIENTOS. }\end{array}$ & & P.POINT: CIMIENTOS \\
\hline 14 MAY & & $\begin{array}{l}\text { PROCESO DE } \\
\text { CÁLCULO }\end{array}$ & $\begin{array}{l}1 \\
2\end{array}$ & $\begin{array}{l}\text { ACT CIM3. REPLANTEO DE LA } \\
\text { CIMENTACIÓN Y SANEAMIENTO. } \\
\text { ACT CIM4. PREDIMENSIONADO Y } \\
\text { ARMADO DE ELEMENTOS. }\end{array}$ & 3 & $\begin{array}{l}\text { P1CI. REPLANTEO DE } \\
\text { CIMIENTOS } \\
\text { P2CI DETALLES CTMIENTOS }\end{array}$ \\
\hline \multirow[t]{2}{*}{$\begin{array}{ll}15 \\
3 \mathrm{JUN}\end{array}$} & $\begin{array}{l}\text { REPASO DE LAA } \\
\text { ASIGNATURA }\end{array}$ & $\begin{array}{l}\text { RESOLUCIÓN DE } \\
\text { DUDAS }\end{array}$ & 1 & $\begin{array}{l}\text { ACT REV2. REVISIÓN DE } \\
\text { NOMENCT.ATURAS }\end{array}$ & & \\
\hline & & & 3 & $\begin{array}{l}\text { ACT F: CURSTIIONARIO FINAL DB } \\
\text { LA ASIGNATURA. }\end{array}$ & & \\
\hline 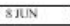 & PRUEBA ESCRITA & & & EVALUACION DE LA DOCENCIA & & \\
\hline
\end{tabular}

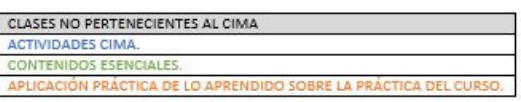

Jornadas de Formación e Innovación Docente del Profesorado | № 2 (2019) Esta obra se distribuye con la licencia Creative Commons Reconocimiento-NoComercial-SinObraDerivada Internacional (CC BY-NC-ND 4.0.) 
Siguiendo la estructura descrita en el diseño de las sesiones propuestas en el aula, el temario de la asignatura se desarrolla en los días previstos tal y como sigue:

\section{Aplicación del CIMA sobre el tema de Estructuras de H.A:}

Los contenidos básicos con los que el estudiante se incorpora al tema son los relativos a la definición y conceptos, tipos de estructuras y elementos que las integran. Los contenidos esenciales que se deben extraer son:

- Diseño de elementos estructurales, pre dimensionado y armado.

- Exigencias de la normativa CTE DB SE y EHE 08.

- Tema Productos: Hormigones, cementos y armaduras.

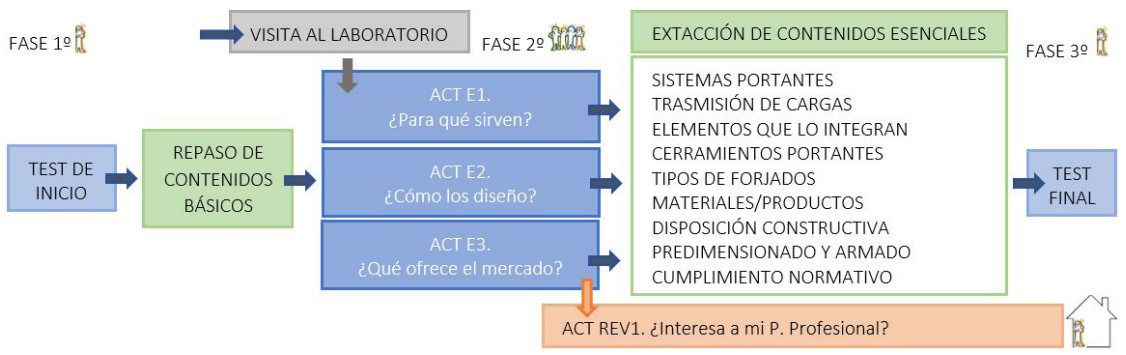

Figura 5. Desarrollo de las sesiones de Estructuras en el aula. Autor

Durante la primera sesión de tema y, tras el repaso de conocimientos y la visita al laboratorio de las sesiones anteriores, las actividades desarrolladas en el aula son:

- Recorrido fotográfico por edificios existentes analizando estructuras y otros capítulos de su construcción, materiales y productos que lo componen y trasmisión de cargas.

- Estudio de caso particular sobre planos y deducción de su esqueleto estructural y trasmisión de cargas.

La segunda sesión desarrolla enlazando las actividades con el repaso de los materiales y productos más 
adecuados según su función en el edificio. A partir de aquí, se desarrollarán otras repartidas en distintas sesiones y que profundizan en el suministro y nomenclaturas sobre cementos, hormigones y armaduras.

\section{Aplicación del CIMA sobre el tema de Forjados Unidireccionales:}

Los contenidos básicos con los que el estudiante se incorpora al tema son los relativos a la definición y conceptos, esquema de cargas, elementos que lo componen o integran las soluciones, incluso un primer acercamiento al replanteo de un forjado, y detalles constructivos. Los contenidos esenciales que se deben extraer son:

- Productos/materiales y diseño. Objetivos.

- Configuración constructiva. Pre dimensionado y armado de elementos.

- Exigencias de la normativa CTE DB SE y EHE 08.

- Revestidos horizontales (Tema Productos: suelos).

- Aplicación práctica de lo aprendido.

Durante la primera sesión de tema se realiza un recorrido fotográfico por un edificio en construcción desarrollándose las siguientes actividades:

- Identificación de elementos que componen un forjado unidireccional de hormigón armado.

- Estudio de un caso particular sobre planos y diseño de secciones indicando materiales y productos que lo componen y su trasmisión de cargas.

En la segunda sesión y, a partir de los detalles del caso anterior, se calcula el canto mínimo del forjado, pre dimensionado y armado de secciones y tipo de vigueta. 


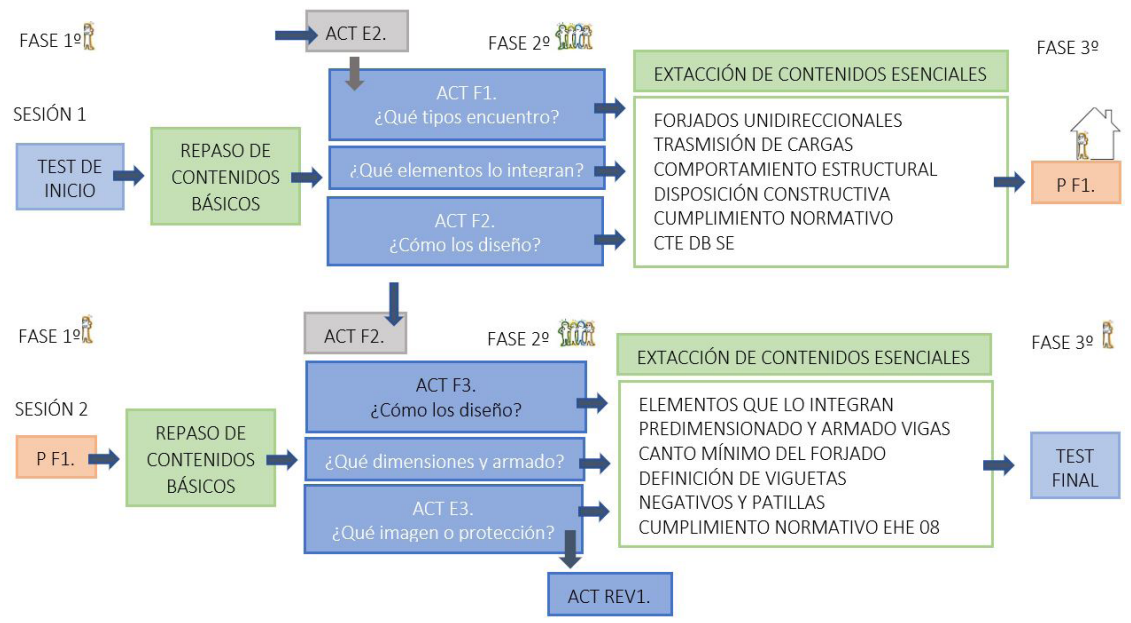

Figura 6. Desarrollo de las sesiones de Forjados en el aula. Autor.

Estas sesiones enlazan con otras actividades que permiten repasar los materiales y productos más comunes como revestimiento del forjado. Estas actividades se resuelven de forma oral y serán los estudiantes los que expliquen las soluciones que defienden.

\section{Aplicación del CIMA sobre el tema de Cerramientos:}

Los contenidos básicos con los que el estudiante se incorpora al tema son los relativos a la definición y conceptos, tipos de cerramientos, peculiaridades de los cerramientos de fábrica de ladrillo, productos/materiales que lo integran y diseño de tipologías de cerramientos. Los contenidos esenciales que se deben extraer son:

- Exigencias de la normativa CTE DB HS y DB HE.

- Revestidos verticales (Tema Productos: cal, yeso, mortero).

- Aplicación práctica de lo aprendido profundizando en el Replanteo de la albañilería y la solución de Puntos singulares y encuentros. 
El tema se inicia con una actividad de repaso sobre las diferencias entre estructura y envolvente en el edificio y, se apoya con un recorrido fotográfico por distintos edificios históricos desarrollándose las siguientes actividades:

- Estudio de un caso particular y repaso de tipos de paramentos verticales, materiales y productos habituales.

- Estudio de un caso particular sobre planos diseñando tipología de acuerdo con la normativa y exigencias de estabilidad y habilidad.

En la segunda sesión, las actividades se centran en edificios deteriorados y lesionados tratando de deducir las soluciones de diseño y construcción más adecuadas. Posteriormente se comprueban las exigencias normativas.

Esta sesión enlaza con otras actividades que permiten repasar los materiales y productos más comunes como revestimiento del edificio. Estas actividades se resuelven de forma oral y serán los estudiantes los que expliquen las soluciones que defienden.

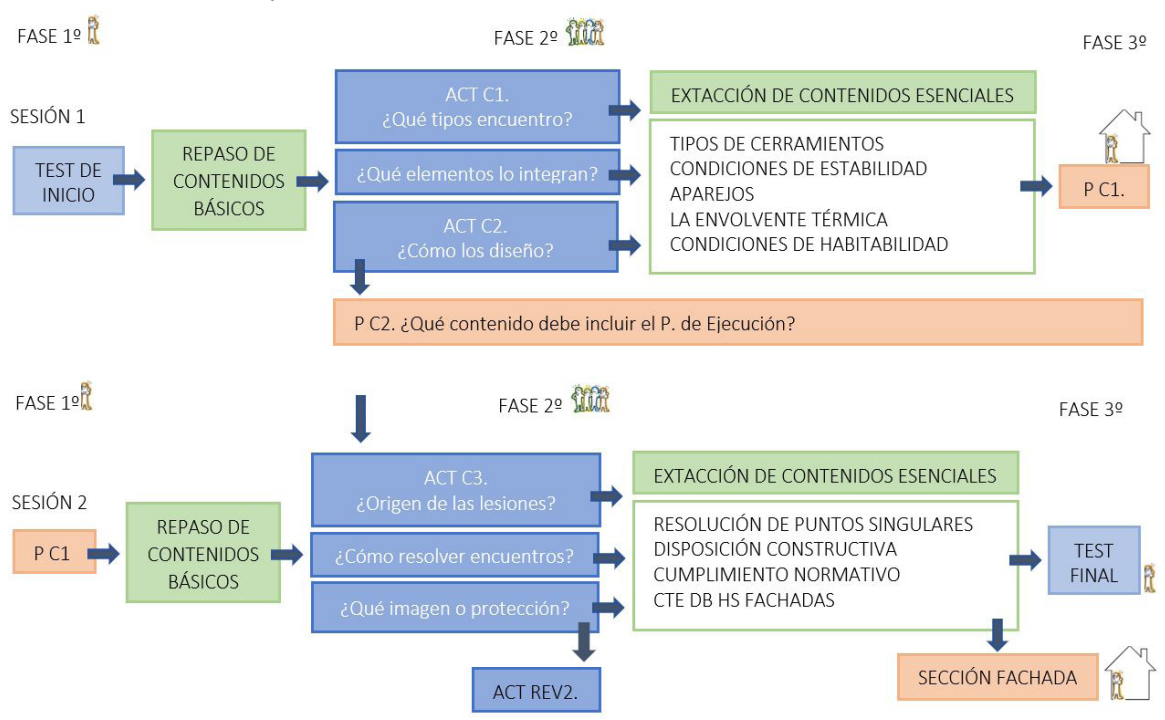

Figura 7. Desarrollo de las sesiones de Cerramientos en el aula. Autor.

Jornadas de Formación e Innovación Docente del Profesorado | № 2 (2019) Esta obra se distribuye con la licencia Creative Commons Reconocimiento-NoComercial-SinObraDerivada 


\section{Aplicación del CIMA sobre el tema de Cubiertas:}

Los contenidos básicos con los que el estudiante se incorpora al tema son los relativos a la definición y conceptos, tipos de cubiertas, elementos que componen o integran el faldón. Productos/materiales y diseño en planta o trazado de la cubierta. Los contenidos esenciales que se deben extraer son:

- Exigencias de la normativa CTE DB HS y DB HE.

- Revestidos horizontales (Tema Productos: impermeabilizantes, techos).

- Aplicación práctica de lo aprendido profundizando en el replanteo de la cubierta y la solución de Puntos singulares y encuentros.

El tema se inicia con una actividad de repaso sobre los tipos de cubiertas que existen se apoya con un recorrido fotográfico por distintos edificios. Posteriormente, se recuerdan los elementos que forman parte del faldón y se desarrollan las siguientes actividades:

- Estudio de un caso particular sobre planos reflexionando sobre el diseño de la cubierta y replanteo de elementos previstos y representación gráfica.

En la segunda sesión, las actividades se centran en edificios con cubiertas deterioradas tratando de deducir las soluciones de diseño y construcción más adecuadas. Posteriormente se comprueban las exigencias normativas.

Esta sesión enlaza con el repaso de las instalaciones que quedarán ocultas en el edificio y las opciones de revestimiento de techos. 


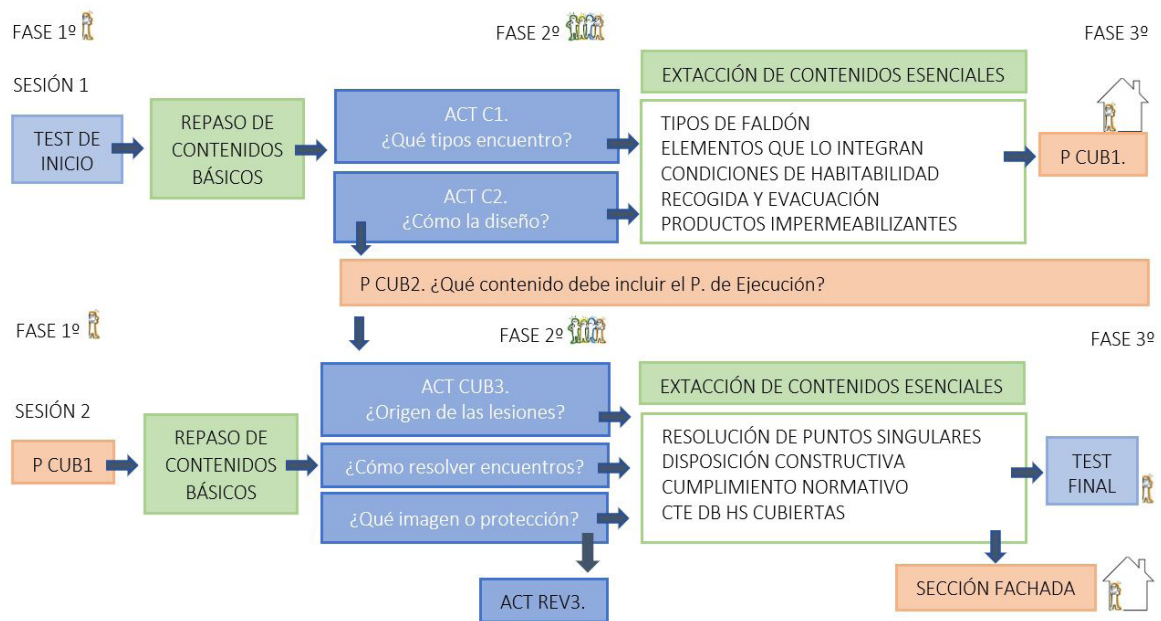

Figura 8. Desarrollo de las sesiones de Cubiertas en el aula. Autor.

\section{Aplicación del CIMA sobre el tema de Terreno y Cimientos:}

Los contenidos básicos con los que el estudiante se incorpora al tema son los relativos al movimiento de tierras y tipos de terreno y excavaciones, la trasmisión de cargas, tipos de cimientos, elementos que lo componen o integran, productos/materiales y diseño en planta. Los contenidos esenciales que se deben extraer son:

- El terreno. El estudio geotécnico.

- Configuración constructiva. Pre dimensionado y armado de elementos.

- Exigencias de la normativa CTE DB SE y EHE 08.

- Aplicación práctica de lo aprendido profundizando en el plano de replanteo de la cimentación y detalles constructivos. 


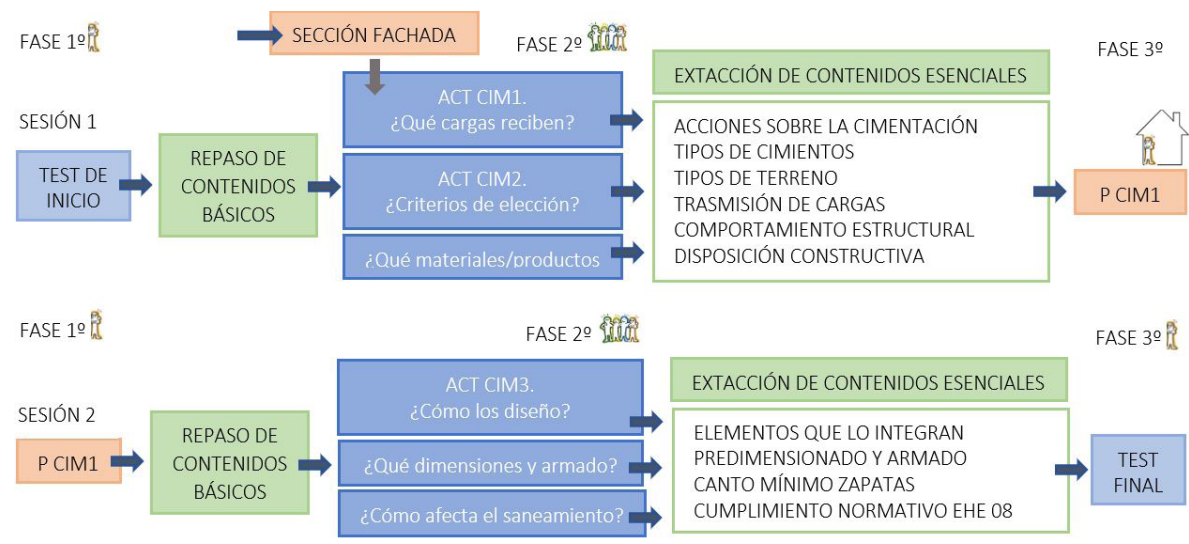

Figura 9. Desarrollo de las sesiones de Cimientos en el aula.

La primera sesión del tema se desarrolla tras el análisis de los tipos de Acciones en la Edificación. Para ello los estudiantes han desarrollado previamente una sección completa del edifico y previsto los materiales y productos que la componen se calculan las cargan que llegan a la cimentación. las actividades posteriores serán:

- Recorrido fotográfico analizando estructuras y trasmisión de cargas al terreno revisando la importancia del Estudio Geotécnico y tipos de cimientos.

- Estudio de un caso particular sobre planos y diseño de la cimentación en planta y sección. Reflexionando sobre su conexión con el saneamiento del edificio.

La sesión 2 se centra en, a partir de los detalles del caso anterior, el cálculo, pre dimensionado y armado de secciones de hormigón.

\section{Resultado y discusión}

Durante el desarrollo de las sesiones y, tras los resultados obtenidos al final del cuatrimestre, se ha tratado de reflexionar sobre distintos aspectos relativos al contenido de las actividades, la organización o la metodología diseñada en tres direcciones (Márquez, 2018): 


\section{Avances y aciertos:}

- Se consolida la idea de partida (propuesta CIMA 2018) sobre controlar la asistencia a partir de las entregas y actividades en clase. Se ahorra tiempo al no pasar lista.

- Durante el desarrollo de las actividades los estudiantes permanecen en el aula. Llama la atención que, aun aquellos que han decidido abandonar la asignatura y la posibilidad de evaluación continua, han continuado en clase realizando las actividades con vistas a una nueva convocatoria.

- Cada actividad del tema ha estado directamente relacionada con la anterior y la posterior (incluyendo preguntas repetidas e incorporando otras de nuevo contenido teórico-práctico) lo que ha permitido dar continuidad a su desarrollo y al aprendizaje final. Asimismo, al inicio de cada tema, las actividades diseñadas estaban directamente relacionadas con el tema anterior lo que ha permitido el repaso periódico y acumulativo de la materia.

- La escalera de aprendizaje aportada en cada actividad ha permitido, al profesor y a el estudiante, conocer la progresión del aprendizaje, así como los contenidos o metodología que presentan dificultades. Se observa, en la figura 10, el descenso en el nivel adquirido en el tema de cimientos, quizás al ser el último tema impartido y someterse a una única actividad, el estudiante no se ha visto favorecido por la repetición de conceptos en otras actividades relacionadas tal y como se ha descrito. 


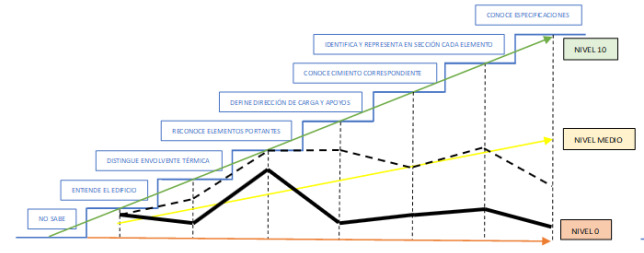

CUESTIONARIO INICIAL DE LA ASIGNATURA

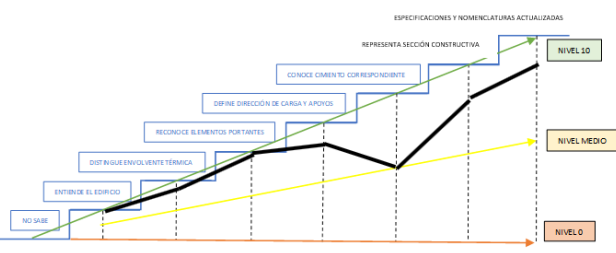

CUESTIONARIO FINAL DE LA ASIGNATURA

- NIVEL DE CONOCIMIENTOS DEL ESTUDIANTE AL DESARROLLAR LA ACTIVIDAD

-... AUTOEVALUACIÓN DEL ESTUDIANTE

Figura 10. Progresión del aprendizaje Construcción 2. Autor.

Respecto a la evaluación de la docencia, los cuestionarios incluidos en cada actividad han permitido extraer conclusiones importantes sobre el desarrollo de cada una de ellas y posibles mejoras a incorporar en el nuevo curso. Asimismo, a final del cuatrimestre se solicita opinión sobre la metodología aplicada. Este cuestionario resume en dos preguntas claves la opinión que aporta el estudiante.

¿En qué parte/ modelo de clase sientes que has aprendido más?:

¿En qué parte te has divertido/implicado más?:

DURANTE LAS EXPLICACIONES TEÓRICAS DEL PROFESOR.

ACTIVIDADES EN EL AULA

REVISANDO PRESENTACIONES DE LA ASIGNATURA

ESTUDIANDO EN CASA

ELABORANDO P.CLASE Y P.CURSO EN CASA

CORRIGIENDO LA PRÁCTICA EN LA PIZARRA.

Otros utilizados (tutoriales, ev3, blog del profesor, etc)

Figura 11. Evaluación de la docencia. Autor.

\section{Incidencias en el desarrollo de la actividad:}

- Los estudiantes revisan de forma autónoma algunas de las presentaciones publicadas de forma oficial. Sin embargo, se quejan del excesivo contenido y desorden de las mismas. 
- Se han aportado las tablas (propuesta CIMA 2018) pero, el estudiante no conoce su origen y, finaliza el curso sin asumir el manejo de la normativa vigente.

- La dinámica de actividades en formato papel ha dificultado la organización prevista de la asignatura (propuesta CIMA 2018) sobre estructurar la asignatura a partir de una secuencia de preguntas planteada previamente.

- No se ha incluido el desarrollo de Mapas de Contenido de cada tema en la pizarra durante las explicaciones (propuesta CIMA 2018) lo que dificulta la visión global del estudiante sobre la asignatura.

- La falta de trabajo en casa impide la continuidad del aprendizaje dificultado la concentración del estudiante en las primeras actividades de cada sesión.

- Por falta de tiempo, este curso no se ha obligado a realizar estregas parciales de la Práctica de Curso prevista en la entrega final. Esto ha supuesto la falta de organización, por parte de muchos estudiantes que, han optado por abandonar en mayo la posibilidad de aprobar por curso (la falta de previsión sobre el volumen de la entrega final y la coincidencia con otras entregas de otras asignaturas ha desbordado la gestión de las mismas).

- No se ha mostrado en el aula exámenes de cursos y convocatorias anteriores según lo previsto en el diseño previo del CIMA. Esta muestra previa aportaría al estudiante la confianza en el contenido estudiado y su adecuación a la evaluación posterior.

- No se han mostrado Proyectos de Ejecución completos en el aula, solo capítulos parciales. El estudiante no ha tenido la visión global de la práctica profesional hasta fin de curso.

- Algunos estudiantes han preferido trabajar de forma individual.

- Los resultados obtenidos en cada tema, sobre la autoevaluación de las actividades, muestran resultados muy distintos al de la evaluación realizada por el 
profesor, lo que hace pensar en una carencia de autocrítica por parte del estudiante.

- No ha existido tiempo suficiente para el debate y discusión de resultados. Asimismo, no se ha dedicado tiempo a exponer una respuesta final correcta y el estudiante la demanda.

\section{Propuestas de mejora (nuevo CIMA 2020)}

- Se hace necesario concretar y reducir las presentaciones de la asignatura para facilitar el seguimiento no presencial del estudiante que. Se ordenarán en función de su necesidad/utilidad en paralelo con el desarrollo de las explicaciones del aula.

- Como alternativa para el aprendizaje en el manejo de la normativa vigente, para próximo curso las actividades indicarán donde localizar las tablas e información adicional y deberá buscarlas y aportarlas el estudiante.

- Para evitar el abandono de la asignatura, se organizarán revisiones semanales y entregas parciales más reducidas que ayuden al estudiante en la gestión del tiempo de la entrega final de la Práctica de curso.

- Dedicar tiempo en el aula a revisar un Proyecto de Ejecución completo y ya visado para un caso real. Como objetivo, relacionar la actividad profesional con el aprendizaje.

- Dedicar tiempo en el aula a revisar exámenes de convocatorias anteriores. Como objetivo, comprobar cómo se les puede evaluar/preguntar en una convocatoria, lo que han hecho en modo práctico.

- Pendiente reflexionar sobre la metodología de aprendizaje de las actividades propuestas, sobre si el estudiante de ir conociendo el proceso de desarrollo de una actividad o la solución correcta al final del proceso para volver sobre los errores. ¿Se podrán incluir los resultados por escrito en vez de dedicar tiempo en la pizarra? 
- Pendiente estudiar técnicas de autocrítica que mejore la valoración y calificación personal del estudiante sobre su nivel de conocimientos como como aprendizaje actitudinal. ¿Deberán recogerse y devolverse las actividades resueltas con mi evaluación para comparar autoevaluación?

- Pendiente reflexionar sobre la utilidad de las actividades como aprendizaje no presencial durante los meses sin docencia para aquellos estudiantes que no han superado la asignatura en primera convocatoria y se presentarán en septiembre.

\section{Conclusiones}

Los resultados que han ido obteniéndose, tras el desarrollo de las actividades y la actitud de los estudiantes durante el transcurso del cuatrimestre, han podido mostrar un acierto importante en la metodología docente aplicada resultando sesiones de 4 horas de duración más dinámicas y amenas que en cursos anteriores. Asimismo, el aprendizaje a partir de problemas ha marcado el ritmo de avance en la materia según la necesidad del estudiante y los resultados muestran que la recepción de contenidos se ha realizado de forma más pausada asimilándose mejor los contenidos con este sistema. Respecto a los objetivos de aprendizaje alcanzados por el estudiante, los resultados muestran que, el desarrollo continuado, acumulativo y repetitivo en las cuestiones que plantean las actividades, ha llevado a un aprendizaje de contenidos esenciales casi inconsciente por parte del estudiante. Al final del curso, el estudiante ha podido resolver, de forma individual, una actividad similar a la prueba final de la asignatura sin esfuerzo adicional.

Respecto a la prueba final de curso para aquellos estudiantes que no han superado la evaluación continua, la sistemática ha permitido una mayor interiorización de los contenidos por lo que, las carencias conceptuales detectadas en la primera convocatoria, han sido menores que en cursos anteriores. 


\section{Referencias bibliográficas}

Bain, K. (2004). Traducción Barberá, O. (2008). Lo que hacen los mejores profesores universitarios. Universidad de Valencia. España: PUV.

Blandón G, B (2018). Propuesta del Nuevo Modelo Didáctico Aplicado en Construcción. Jornadas de Formación e Innovación Docente del Profesorado. Universidad de Sevilla. http://dx.doi.org/10.12795/JDU.2018.i01.03

Bur, Aníbal (2012). El Aprendizaje Basado en Problemas (ABP) en el aula universitaria. XX Jornadas de Reflexión Académica en Diseño y Comunicación Facultad de Diseño y Comunicación. Universidad de Palermo. ISSN: 1668-1673

Finkel, D. Traducción Barberá, O. (2008). Dar clases con la boca cerrada. Valencia. España: Universidad de Valencia. Libro de resúmenes del 1 Congreso Internacional de In- novación y Tendencias Educativas. INNTED (2017). Innovación y Tendencias Educativas. Sevilla. España: Universidad de Sevilla.

Márquez Guerrero, C. (2018). La metodología de "Aprendizaje basado en problemas" para la enseñanza de la asignatura "Economía mundial": el mito del Desarrollo y del Crecimiento Económico Infinito. Jornadas de Formación e Innovación Docente del Profesorado. Universidad de Sevilla. http://dx.doi.org/10.12795/JDU.2018. i01.23

Porlán, R. (2017). Enseñanza Universitaria. Como mejorarla. Universidad de Sevilla. España: Ediciones Morata.

Jornadas de Formación e Innovación Docente del Profesorado | № 2 (2019) Esta obra se distribuye con la licencia Creative Commons Reconocimiento-NoComercial-SinObraDerivada Internacional (CC BY-NC-ND 4.0.) 